\title{
L'expérimentation sur le porc chinois en France II. - Performances de production en croisement avec les races européennes
}

\author{
C. LEGAULT, P. SELLIER, J.C. CARITEZ *, P. DANDO ** et J. GRUAND *** \\ avec la collaboration de C. Dupont*, J. Gogué, C. Guérin \\ et Marie-Reine Perretant \\ I.N.R.A., Station de Génétique quantitative et appliquée, \\ Centre de Recherches zootechniques, F 78350 Jouy-en-Josas \\ * Domaine expérimental du Magneraud, F 17700 Surgères \\ ** Domaine de Galle, F 18520 Avord \\ *** Station expérimentale de Sélection porcine, F 86480 Rouillé
}

\begin{abstract}
Résumé
L'objet de cet article est l'analyse des données de croissance et de carcasse recueillies de 1980 à 1982 sur des porcs issus de croisements à 2 et 3 voies impliquant les 3 races chinoises introduites en France en 1979 (Meishan : MS, Jiaxing : JX, Jinhua : JH) et diverses races européennes (Large White : LW, Landrace Français : LF, Piétrain : $\mathrm{P}$, Landrace Belge : LB). Chacune des races chinoises n'était représentée que par 3 animaux fondateurs ( 1 mâle et 2 femelles). Quatre comparaisons distinctes ont été réalisées. Le contrôle de croissance (de $26-29 \mathrm{~kg}$ à $93-100 \mathrm{~kg}$ selon la comparaison) a été réalisé, en alimentation à volonté, sur un total de 1085 animaux, dont 887 ont fait l'objet d'une évaluation de la qualité de carcasse.

La comparaison $n^{\circ} 1$ concerne les croisements $F_{1}$ entre des verrats MS, JX ou JH et des truies LW ou LF. Les moyennes des porcs $1 / 2$ MS $(n=49), 1 / 2 \quad J X(n=40)$ et $1 / 2 \mathrm{JH}(\mathrm{n}=23)$ ont été respectivement : 756,767 et $660 \mathrm{~g} / \mathrm{j}$ pour le gain moyen quotidien (GMQ) ; 76,0, 76,4 et 77,4 p. 100 pour le rendement en carcasse (REND) ; 38,8, 38,8 et 36,6 p. 100 pour le pourcentage de muscle estimé dans la carcasse (PMEC, référence C.E.E.). La comparaison $n^{\circ} 2$ concerne les croisements $F_{1}$ entre des verrats MS ou JX et des truies $P$ et la race pure Piétrain. Les moyennes des porcs MS $\times P(n=46), J X \times P$ $(\mathrm{n}=45)$ et $\mathrm{P} \times \mathrm{P}(\mathrm{n}=60)$ ont été respectivement pour les variables GMQ, REND et PMEC : 509, 503 et $427 \mathrm{~g} / \mathrm{j} ; 77,4,77,3$ et 80,6 p. $100 ; 45,7,45,6$ et 54,4 p. 100 . L'ensemble des comparaisons $\mathrm{n}^{\circ \mathrm{s}} 3$ et 4 correspond à un croisement diallèle incomplet avec 2 races de pères (LB et $L W$ ) et 8 types génétiques de mères $F_{1}$. Les produits de ces croisements ont été regroupés en 4 classes : $1 / 4$ MS (mères MS $\times \mathrm{LW}, \mathrm{LW} \times \mathrm{MS}$ ou $\mathrm{MS} \times \mathrm{LF}$ ), $1 / 4 \mathrm{JX}$ (mères $\mathrm{JX} \times \mathrm{LW}$ ou $\mathrm{JX} \times \mathrm{LF}$ ), $1 / 4 \mathrm{JH}$ (mères $\mathrm{JH} \times \mathrm{LW}$ ou $\mathrm{JH} \times \mathrm{LF}$ ) et "témoin " européen (mères LF $\times$ LW). Les moyennes des porcs $1 / 4$ MS $(n=317)$, $1 / 4 \mathrm{JX}(\mathrm{n}=306), 1 / 4 \mathrm{JH}(\mathrm{n}=114)$ et «témoins》 $(\mathrm{n}=85)$ ont été respectivement : $790,754,700$ et $818 \mathrm{~g} / \mathrm{j}$ pour GMQ $3,63,3,74,3,79$ et $3,40 \mathrm{~kg}$ aliment $/ \mathrm{kg}$ gain pour l'indice de consommation; 77,9, 78,4,79,4 et 78,3 p. 100 pour REND; 95,8, 97,9, 95,3 et $97,0 \mathrm{~cm}$ pour la longueur de carcasse; $10,47,10,54,10,65$ et $11,20 \mathrm{~kg}$ pour le poids de longe ; $5,21,5,24,5,81$ et $4,72 \mathrm{~kg}$ pour le poids de bardière ; 45,6, 45,1, 43,9 et 49,1 p. 100 pour PMEC; $86,3,86,1,86,0$ et 85,8 points pour l'indice de qualité de la viande. Pour la marge brute à l'engraissement, le désavantage des animaux $1 / 4 \mathrm{MS}, 1 / 4 \mathrm{JX}$ et
\end{abstract}


$1 / 4 \mathrm{JH}$ vis-à-vis du " témoin» européen a été chiffré à environ 54,74 et 93 francs par porc, respectivement.

La réduction de la marge brute à l'engraissement chez les produits $1 / 4$ chinois est à confronter à l'avantage apporté par la meilleure productivité numérique de leurs mères $1 / 2$ chinoises, qui se traduit par une diminution sensible du prix de revient du porcelet au sevrage (cf. partie I de l'étude : Génét. Sél. Evol., 15, 225-240). A ce stade de nos investigations, il apparaît que, comparé à l'utilisation de truies européennes, le recours à des truies $1 / 2$ chinoises donne un résultat économique global voisin de l'équilibre pour les $1 / 2 \mathrm{MS}$, légèrement défavorable pour les $1 / 2 \mathrm{JX}$ et très nettement défavorable pour les $1 / 2 \mathrm{JH}$.

Mots clés : Porcins, croisement, races chinoises, croissance, carcasse.

\section{Summary}

Experiments with Chinese pigs in France.

\section{Productive performance in crosses with European breeds}

The aim of this paper is to analyse growth and carcass data recorded from 1980 to 1982 on pigs from 2- and 3-way crosses involving the 3 Chinese breeds imported into France in 1979 (Meishan : MS, Jiaxing : JX, Jinhua : JH) and various European breeds (Large White : LW, French Landrace : FL, Pietrain : P, Belgian Landrace : BL). Each Chinese breed was represented by only 3 founder animals ( 1 boar and 2 gilts). Four distinct comparisons were carried out. Growth records (from $26-29 \mathrm{~kg}$ to $93-100 \mathrm{~kg}$ according to the comparison) were obtained on ad libitum feeding on a total of 1085 pigs, among which 887 were submitted to carcass evaluation.

Comparison no. 1 deals with $F_{1}$ crosses between MS, JX or JH sires and LW or FL dams. Means of $1 / 2$ MS $(n=49), 1 / 2 \mathrm{JX}(\mathrm{n}=40)$ and $1 / 2 \mathrm{JH}$ pigs $(\mathrm{n}=23)$ were respectively : 756, 767 and $660 \mathrm{~g} /$ day in average daily gain (ADG); 76.0, 76.4 and 77.4 p. 100 in killing out percentage (KOP); 38.8, 38.8 and 36.6 p. 100 in estimated carcass lean percentage (ECLP, E.E.C. reference). Comparison no. 2 deals with $F_{1}$ crosses between MS or JX sires and $P$ dams and the pure Pietrain breed. Means of MS $\times P(n=46)$, $J X \times P(n=45)$ and $P \times P$ pigs $(n=60)$ were respectively : 509,503 and $427 \mathrm{~g} /$ day in ADG : 77.4, 77.3 and 80.6 p. 100 in KOP; $45.7,45.6$ and 54.4 p. 100 in ECLP. Comparisons nos. 3 and 4 correspond to an incomplete diallel cross with 2 breeds of sire (BL or LW) and 8 genetic types of $F_{1}$ dams. Progeny of these crosses were pooled in 4 classes : $1 / 4 \mathrm{MS}(\mathrm{MS} \times \mathrm{LW}, \mathrm{LW} \times \mathrm{MS}$ and $\mathrm{MS} \times \mathrm{FL}$ dams $), 1 / 4 \mathrm{JX}(\mathrm{JX} \times \mathrm{LW}$ or $\mathrm{JX} \times \mathrm{FL}$ dams), $1 / 4 \mathrm{JH}(\mathrm{JH} \times \mathrm{LW}$ or $\mathrm{JH} \times \mathrm{FL}$ dams $)$, and European "control " $(\mathrm{FL} \times \mathrm{LW}$ dams). Means of $1 / 4$ MS $(\mathrm{n}=317), 1 / 4 \mathrm{JX}(\mathrm{n}=306), 1 / 4 \mathrm{JH} \quad(\mathrm{n}=114)$ and " control $\gg$ pigs $(n=85)$ were respectively : $790,754,700$ and $818 \mathrm{~g} /$ day in ADG ; $3.63,3.74,3.79$ and $3.40 \mathrm{~kg}$ feed $/ \mathrm{kg}$ gain in food conversion ratio; $77.9,78.4,79.4$ and 78.3 p. 100 in KOP ; 95.8, 97.9, 95.3 and $97.0 \mathrm{~cm}$ in carcass length; 10.47, 10.54, 10.65 and 11.20 in weight of loin; $5.21,5.24,5.81$ and $4.72 \mathrm{~kg}$ in weight of backfat ; 45.6, 45.1, 43.9 and 49.1 p. 100 in ECLP ; 86.3, 86.1, 86.0 and 85.8 points in meat quality index. In gross margin from fattening, the disadvantage of $1 / 4 \mathrm{MS}, 1 / 4 \mathrm{JX}$ and $1 / 4 \mathrm{JH}$ pigs, as compared to European "control » pigs, was estimated at around 54, 74 and $93 \mathrm{FF}$ per pig, respectively.

The reduction of the gross margin from fattening in 1/4 Chinese pigs is to be compared to the advantage brought by the improved numerical productivity of their half-Chinese dams, which results in a substantially lowered production cost of the weaned piglet (cf. part I of this study : Génét. Sél. Evol., 15, 225-240). At this stage of our investigations, it appears that the use of half-Chinese dams, as compared to European dams, provides a total economic result which is near the equilibrium for $1 / 2 \mathrm{MS}$, slightly unfavourable for $1 / 2 \mathrm{JX}$, and strongly unfavourable for $1 / 2 \mathrm{JH}$ dams.

Key words : Pigs, crossbreeding, Chinese breeds, growth, carcass. 


\section{Introduction}

L'analyse comparative des performances de reproduction de 3 races locales chinoises récemment introduites en France (Meishan, Jiaxing et Jinhua), de 2 races européennes (Large White et Landrace Français) et de leurs croisements attire l'attention sur le rôle déterminant que sont susceptibles de tenir certaines races chinoises dans l'amélioration génétique de la productivité numérique des truies (Legault \& CARITEZ, 1983). En particulier, le croisement entre 2 des races chinoises étudiées (Meishan et Jiaxing) et les 2 races européennes peut conduire à la mise à la reproduction d'une femelle $F_{1}$ plus précoce (âge à la première mise bas avancé d'au moins 1 mois), plus économe (consommation annuelle d'aliment concentré réduite d'au moins $100 \mathrm{~kg}$ ) et surtout plus productive ( 5 à 8 porcelets supplémentaires sevrés chaque année) : il peut en résulter une diminution du prix de revient du porcelet au sevrage de l'ordre de 40 à 60 francs. Bien entendu, cet avantage doit être confronté à la dégradation prévisible de la marge brute à l'engraissement chez les porcs provenant de croisements à base de races chinoises.

L'objet de cet article est précisément une tentative de synthèse des résultats recueillis de 1980 à 1982 dans 3 troupeaux expérimentaux de l'I.N.R.A. sur les performances de croissance et de carcasse des produits terminaux issus de divers plans de croisement impliquant les 3 races chinoises. Des résultats préliminaires ont été publiés sur ce sujet par LEgAult et al. (1982) et Gianola et al. (1982). La plus grande attention sera accordée ici d'une part au mérite comparé des races chinoises Meishan, Jiaxing et Jinhua, d'autre part à la comparaison des produits terminaux issus de truies $1 / 2$ chinoises et des produits terminaux «témoins » issus de truies européennes.

\section{Matériel et méthodes}

\section{A. Animaux et dispositifs expérimentaux}

La présente expérimentation fait suite à l'importation en 1979 de 3 reproducteurs ( 1 mâle et 2 femelles) de chacune des 3 races chinoises Meishan (MS), Jiaxing (JX) et Jinhua $(\mathrm{JH})$, dont l'origine géographique et les caractéristiques zootechniques ont été rappelées dans la première partie de cette étude (LEgault \& CARITEZ, 1983) et sont décrites par Wu \& Zhang (1982), Zhang et al. (1983) et Cheng (1983, 1984). Au total, de 1980 à 1982, 1085 animaux ont été soumis à un contrôle de croissance et 887 d'entre eux ont fait l'objet d'une évaluation de la qualité de la carcasse.

Quatre comparaisons distinctes ont été réalisées. Les plans d'accouplement, les effectifs de reproducteurs mâles et femelles utilisés et de descendants contrôlés sont précisés dans le tableau 1 pour les comparaisons $n^{\text {os }} 1$ et 2 (porcs $1 / 2$ chinois) et dans le tableau 2 pour les comparaisons $n^{\text {os }} 3$ et 4 (porcs $1 / 4$ chinois).

La comparaison $n^{\circ} 1$ porte sur des porcs appartenant aux 6 types génétiques résultant de l'accouplement entre les 3 verrats fondateurs MS, JX, JH et des truies primipares des races européennes Large White (LW) et Landrace Français (LF). Cette comparaison s'est déroulée en 1980 au Domaine expérimental du Magneraud (Surgères, Charente-Maritime), lieu d'implantation des animaux de race chinoise. 


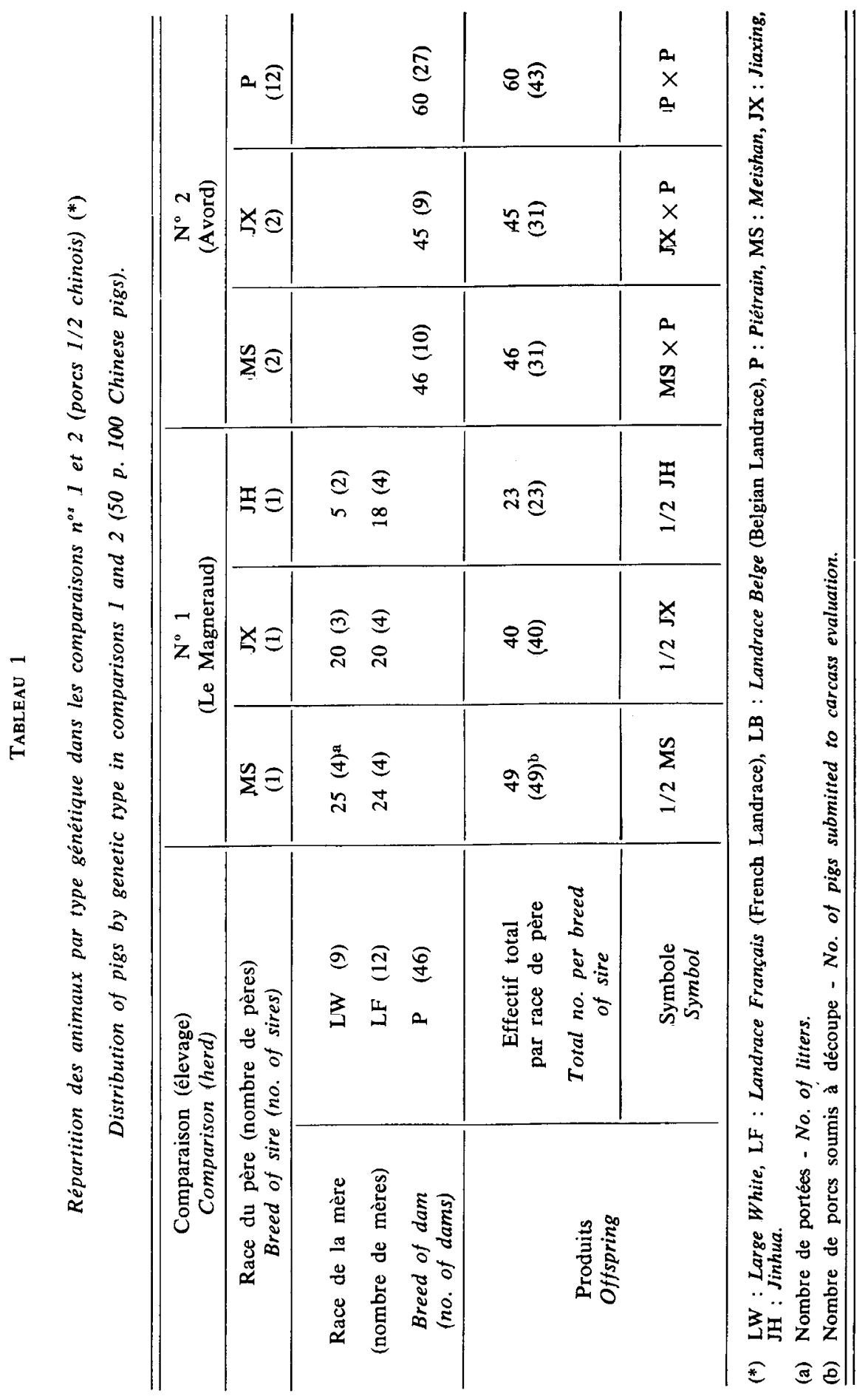




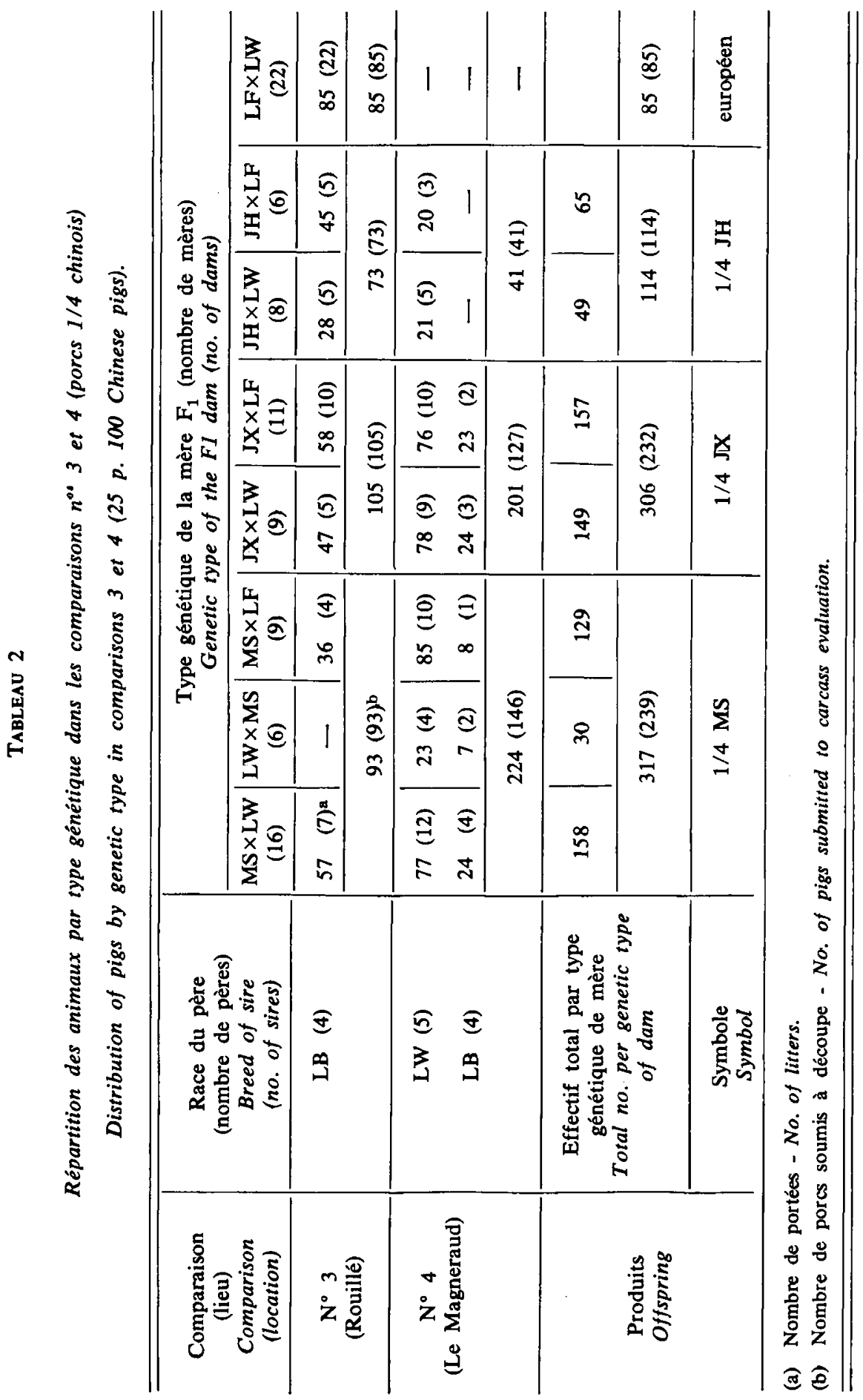


La comparaison $\mathrm{n}^{\circ} 2$, qui s'est déroulée au Domaine expérimental de Galle (Avord, Cher), porte sur 91 porcs MS $\times \mathrm{P}$ ou JX $\times \mathrm{P}$, issus du croisement entre des verrats Meishan ou Jiaxing et des truies Piétrain (P), et sur 60 porcs " témoins » de race pure Piétrain $(\mathrm{P} \times \mathrm{P})$.

Les données relatives aux animaux $1 / 4$ chinois ont été recueillies simultanément (en 1981-1982) dans 2 troupeaux expérimentaux de l'I.N.R.A. : la Station expérimentale de Sélection porcine (S.E.S.P.) située à Rouillé (Vienne) pour la comparaison $\mathrm{n}^{\circ} 3$, et le Domaine du Magneraud pour la comparaison $\mathrm{n}^{\circ} 4$. A Rouillé, le contrôle de croissance, de consommation d'aliment et d'abattage a porté en 2 bandes successives sur un total de 356 animaux, à savoir 271 porcs $1 / 4$ chinois, nés au Magneraud et transférés à Rouillé vers le poids de $20 \mathrm{~kg}$, et 85 porcs «témoins » européens. Comme indiqué au tableau 2, ces 356 porcs sont issus de verrats Landrace Belge (LB) en service à la Station expérimentale d'insémination artificielle (S.E.I.A.) de Rouillé et dont la semence a été utilisée sur des truies MS $\times$ LW ou MS $\times$ LF (porcs $1 / 4 \mathrm{MS}$ ), $\mathrm{JX} \times \mathrm{LW}$ ou $\mathrm{JX} \times \mathrm{LF}$ (porcs $1 / 4 \mathrm{JX}$ ), $\mathrm{JH} \times \mathrm{LW}$ ou $\mathrm{JH} \times \mathrm{LF}$ (porcs $1 / 4 \mathrm{JH}$ ) et sur des truies LF $\times$ LW (porcs de type génétique européen). Au Magneraud, le contrôle de croissance a été réalisé en 3 bandes sur un total de 466 porcs (dont 314 soumis à la découpe normalisée de la carcasse). Ces porcs sont issus de verrats LB ou LW de la S.E.I.A. et de truies $1 / 2$ chinoises de 7 types génétiques différents, décrits au tableau 2. Ce sont les 4 mêmes verrats LB qui ont été utilisés par insémination artificielle dans les comparaisons $\mathrm{n}^{\circ} 3$ et 4 et les 36 truies $1 / 2$ chinoises qui ont eu des descendants contrôlés à Rouillé ont également eu des descendants contrôlés au Magneraud : il existe donc une connexion étroite entre les 2 comparaisons, dont l'ensemble constitue un croisement diallèle incomplet avec 2 races paternelles et 8 types génétiques maternels (cf. tabl. 2).

\section{B. Modalités des contrôles et variables étudiées}

Les contrôles se sont déroulés de façon similaire, à quelques variantes près, dans les 4 comparaisons.

Vers le poids de $25 \mathrm{~kg}$, les porcelets (femelles et mâles castrés) ont été répartis par loges d'environ 10 porcs de même type génétique. Pendant le contrôle de croissance, ils étaient alimentés à volonté (nourrisseur automatique) avec un aliment à 16,5 p. 100 de matières azotées totales et $3200 \mathrm{Kcal}$ d'énergie digestible par $\mathrm{kg}$. Les bâtiments sont fermés, isolés et non chauffés au Magneraud (comparaisons 1 et 4) alors qu'ils sont semi-ouverts dans les 2 autres élevages (comparaisons 2 et 3). En fin de contrôle, les animaux étaient pesés après une mise à jeun de $24 \mathrm{~h}$ et abattus à un poids vif voisin de $100 \mathrm{~kg}$ au Magneraud et à Rouillé et de $93 \mathrm{~kg}$ au Domaine de Galle.

Les animaux des 2 bandes de contrôle de la comparaison $\mathrm{n}^{\circ} 3$ ont été répartis en 10 «blocs» de 4 loges adjacentes, l'une des loges étant occupée par des animaux " témoins " et chacune des 3 autres loges étant occupée par des animaux 1/4 MS, $1 / 4 \mathrm{JX}$ et $1 / 4 \mathrm{JH}$. La consommation d'aliment était contrôlée par loge et par conséquent par type génétique de $30 \mathrm{~kg}$ à l'abattage.

Le lendemain de l'abattage, il a été procédé à la pesée de la carcasse entière (avec tête, pieds et panne), à la mesure de la longueur de carcasse (entre l'atlas et le bord antérieur de la symphyse pubienne), à la mesure de l'épaisseur de lard sur la fente 
saggitale au niveau de la dernière vertèbre lombaire ( « rein "), de la dernière vertèbre dorsale ( « dos ») et de la dernière vertèbre cervicale (« cou »), à la découpe parisienne normalisée d'une demi-carcasse (Ollivier, 1970), ainsi qu'à des mesures de qualité de la viande ( $\mathrm{pH}$ ultime, réflectance, temps d'imbibition) sur différents muscles (Sellier et al., 1984). Les variables considérées dans le présent article sont précisées dans les tableaux de résultats. Parmi elles figurent 2 variables « synthétiques » : un estimateur de la teneur en muscle de la carcasse et un indice de qualité de la viande.

Le pourcentage de muscle dans la carcasse avec tête, pieds et panne (référence C.E.E.) a été estimé d'après l'équation établie par Pommeret \& NAveau (1979) et basée sur les résultats de la découpe parisienne normalisée :

$$
\mathrm{Y}_{\mathrm{CEE}}=-0,75+0,80 \mathrm{~J}+1,06 \mathrm{~L}+0,48 \mathrm{P}_{\mathrm{o}}-0,50 \mathrm{~B}-0,66 \mathrm{P}_{\mathrm{A}}
$$

où $\mathrm{J}, \mathrm{L}, \mathrm{P}_{\mathrm{o}}, \mathrm{B}$ et $\mathrm{P}_{\mathrm{A}}$ sont respectivement les pourcentages de jambon, de longe, de poitrine, de bardière et de panne dans la demi-carcasse soumise à découpe.

L'indice de qualité de la viande (IQV), conçu comme un prédicteur du rendement technologique de la fabrication du jambon de Paris $(\mathrm{R}=0,72)$ et établi par JAcouet et al. (1984), est de la forme :

$$
\mathrm{IQV}=53,63+0,1734 \mathrm{IMB}+5,9019 \mathrm{PHU}-0,0092 \mathrm{REF}
$$

où IMB est le temps d'imbibition du muscle Biceps femoris (en dizaines de secondes), PHU le $\mathrm{pH}$ ultime du muscle Adductor femoris et REF la réflectance du muscle Biceps femoris (échelle 0-1000).

\section{Analyse statistique}

Les données concernant les animaux $1 / 2$ chinois ont été analysées séparément pour les comparaisons $n^{\circ} 1$ (Le Magneraud) et $n^{\circ} 2$ (Avord). En revanche, les données relatives aux animaux $1 / 4$ chinois, recueillies dans 2 troupeaux différents (Le Magneraud et Rouillé), ont été analysées ensemble. Dans toutes ces analyses, les moyennes des types génétiques mis en comparaison ont été estimées par la méthode des moindres carrés appliquée à des modèles d'analyse de variance à effets fixés prenant en compte, selon la comparaison et le groupe de variables, des facteurs de variation différents.

Dans la comparaison $\mathrm{n}^{\circ} 1$, le modèle d'analyse comprend les effets de la race du père ( 3 niveaux, correspondant aux 3 verrats fondateurs : $\mathrm{MS}, \mathrm{JX}, \mathrm{JH}$ ), de la race de la mère ( 2 niveaux : LW et LF), du sexe ( 2 niveaux : femelles et mâles castrés), ainsi que la régression linéaire sur le poids initial du contrôle de croissance (pour le gain moyen quotidien) ou sur le poids vif d'abattage (pour les caractères de carcasse). Dans la comparaison $\mathrm{n}^{\circ} 2$, une seule race de mère est présente (Piétrain) et le modèle d'analyse inclut les effets de la race du père ( 3 niveaux : $M S, J X, P$ ), du sexe (2 niveaux), au bâtiment ( 3 niveaux), de l'interaction race du père $\times$ sexe, ainsi que la covariable poids initial ou poids d'abattage, comme ci-dessus. Dans les 2 comparaisons, l'effet de la date d'abattage est inclus dans le modèle d'analyse des variables relatives à la qualité de la viande.

Comme indiqué plus haut, les données des comparaisons $\mathrm{n}^{\circ \mathrm{s}} 3$ et 4 , relatives aux porcs $1 / 4$ chinois, sont regroupées pour l'analyse. Dans une première analyse, les termes du modèle sont les suivants : 
- l'élevage (2 niveaux : Rouillé et Le Magneraud);

- la bande de contrôle intra-élevage ( 5 niveaux au total);

- la race du père ( 2 niveaux : LB et $L W)$;

- le père intra-race de père ( 9 niveaux au total);

- le sexe ( 2 niveaux : femelles et mâles castrés);

- le type génétique de la mère ( 7 niveaux : MS $\times \mathrm{LW}$ ou $\mathrm{LW} \times \mathrm{MS}, \mathrm{MS} \times \mathrm{LF}$, $\mathrm{JX} \times \mathrm{LW}, \mathrm{JX} \times \mathrm{LF}, \mathrm{JH} \times \mathrm{LW}, \mathrm{JH} \times \mathrm{LF}$ et $\mathrm{LF} \times \mathrm{LW})$;

- l'interaction sexe $\times$ type génétique de la mère ;

- la régression linéaire sur le poids à la mise en contrôle (gain moyen quotidien) ou sur le poids vif d'abattage (caractères de carcasse).

Pour les variables de qualité de la viande, l'effet de la date d'abattage (au total 29 niveaux) remplace l'effet de la bande de contrôle dans le modèle d'analyse.

Les résultats de cette première analyse ont montré que la différence entre les 2 races grand-maternelles européennes (LW et $L F$ ) était non significative pour la quasi-totalité des variables, et ceci quelle que soit la race chinoise utilisée comme " partenaire ». Ce fait a conduit, pour simplifier la présentation, à regrouper les types génétiques maternels en 4 classes $(1 / 2 \mathrm{MS}, 1 / 2 \mathrm{JX}, 1 / 2 \mathrm{JH}$ et européen) et à réaliser une seconde analyse, dont le modèle inclut les mêmes termes que ci-dessus, mais avec seulement 4 niveaux pour le type génétique de la mère.

L'indice de consommation et la consommation moyenne journalière d'aliment n'ont pu être analysés que sur les données de la comparaison $n^{\circ} 3$, réalisée à Rouillé avec une seule race de père (LB). L'analyse porte sur les valeurs calculées par loge (40 loges au total), selon un modèle incluant les effets du type génétique de la mère (4 niveaux ; 1/2 MS, 1/2 JX, 1/2 JH, européen), de la bande de contrôle (2 niveaux), du «bloc » intra-bande de contrôle (au total 10 niveaux), les régressions linéaires sur les poids moyens en début et en fin de contrôle et sur le « sex-ratio " des porcs de la loge.

Dans l'ensemble des analyses, des contrastes linéaires entre les moyennes des moindres carrés ont été calculés afin d'établir à l'aide du test $\mathrm{t}$ de Student le degré de signification statistique des différences entre types génétiques pris 2 à 2 .

Les calculs ont été réalisés au Centre de Traitement de l'Information général (C.T.I.G.) de Jouy-en-Josas, à l'aide de divers programmes disponibles dans le logiciel SAS ( Statistical Analysis System»).

\section{Résultats}

Afin de ne pas alourdir la présentation et de faciliter l'interprétation, nous ne rapportons ici que les résultats ayant trait aux 2 principaux objectifs de l'étude, à savoir la comparaison des 3 races chinoises entre elles et la comparaison des produits terminaux $1 / 4$ chinois et des produits terminaux " témoins " d'origine européenne, pour les performances de croissance et de carcasse. 


\section{A. Porcs $1 / 2$ chinois}

Nous donnons dans le tableau 3 les moyennes des moindres carrés pour chacune des 3 races paternelles de la comparaison $n^{\circ} 1(\mathrm{MS}, \mathrm{JX}, \mathrm{JH})$ et de la comparaison $\mathrm{n}^{\circ} 2$ (MS, JX, P). Cette présentation simplifiée est, pour toutes les variables concernées, justifiée dans la comparaison $n^{\circ} 1$ par l'absence d'interaction race de père $\times$ race de mère et, dans les 2 comparaisons, par l'absence d'interaction sexe $\times$ type génétique.

\section{Comparaison $n^{\circ} 1$}

La croissance des animaux est relativement rapide puisque le poids d'abattage de $99 \mathrm{~kg}$ est atteint respectivement à l'âge de 159,157 et 167 jours chez les $1 / 2 \mathrm{MS}$, les $1 / 2 \mathrm{JX}$ et les $1 / 2 \mathrm{JH}$. Le retard de croissance des $1 / 2 \mathrm{JH}$ sur les 2 autres types génétiques est significatif $(\mathrm{P}<0,01)$. En revanche, le rendement à l'abattage des porcs $1 / 2 \mathrm{JH}$ est plus élevé que celui des 2 autres types génétiques $(77,4$ p. 100 contre 76,0 et 76,4 respectivement pour les $1 / 2$ MS et les $1 / 2$ JX). La qualité de la carcasse est dans l'ensemble très médiocre sur le plan du rapport muscle/gras. Pour le pourcentage estimé de muscle dans la carcasse (avec tête), la descendance du verrat $\mathrm{JH}$ est significativement inférieure à celle des 2 autres verrats : $36,6 \mathrm{p} .100$ pour les $1 / 2 \mathrm{JH}$ contre 38,8 p. 100 pour les $1 / 2$ MS et les $1 / 2$ JX. Pour l'épaisseur moyenne de lard dorsal sur la carcasse, les $1 / 2$ MS $(37,7 \mathrm{~mm})$ occupent une position intermédiaire entre les 1/2 JX $(34,3 \mathrm{~mm})$ et les $1 / 2 \mathrm{JH}(41,6 \mathrm{~mm})$, toutes les différences étant significatives. Notons également que les carcasses des descendants du verrat JX sont significativement plus longues que celles des descendants des verrats MS et JH. Pour le $\mathrm{pH}$ ultime et le pouvoir de rétention d'eau de la viande, on n'observe pas de différence significative entre les 3 races paternelles. Toutefois, l'indice de qualité de la viande (IQV) est significativement plus élevé chez les $1 / 2 \mathrm{JH}$ que chez les $1 / 2 \mathrm{JX}$, du fait d'une réflectance plus faible.

\section{Comparaison $n^{\circ} 2$}

Les données recueillies à Avord permettent de situer 2 des races chinoises (MS et $\mathrm{JX)}$ par rapport à la race Piétrain $(\mathrm{P})$, utilisée comme race maternelle et comme "témoin ». La vitesse de croissance est significativement plus rapide chez les porcs $\mathrm{MS} \times \mathrm{P}$ et $\mathrm{JX} \times \mathrm{P}$ que chez les porcs $\mathrm{P} \times \mathrm{P}$ et le poids d'abattage $(93 \mathrm{~kg})$ est atteint respectivement 26 et 21 jours plus tôt chez les MS $\times P$ et les $\mathrm{JX} \times \mathrm{P}$ que chez les «témoins" $\mathrm{P} \times \mathrm{P}$.

Le rendement à l'abattage est plus élevé $(+3,2$ à 3,3 points de pourcentage) chez les "témoins " Piétrain que chez les animaux $1 / 2$ chinois, la différence étant hautement significative $(P<0,01)$. La longueur de la carcasse est significativement plus faible chez les porcs $P \times P(89,8 \mathrm{~cm}$ contre $91,7 \mathrm{~cm})$. Le pourcentage estimé de muscle dans la carcasse est d'environ 9 points plus élevé chez les « témoins " Piétrain $(54,4$ p. 100 contre 45,7 p. 100 chez les MS $\times P$ et 45,6 p. 100 chez les JX $\times P$ ). Outre le fait que l'épaisseur du lard dorsal sur la carcasse est significativement plus élevée chez les $1 / 2$ chinois et qu'il n'y a pas de différence entre les porcs MS $\times P$ et $\mathrm{fX} \times \mathrm{P}$, on note une grande divergence quant à la différence d'épaisseur de lard sur la fente entre les sites anatomiques du « rein » et du «dos»: la différence (lard au " rein" - lard au « dos») est positive $(+2 \mathrm{~mm})$ chez les sujets $1 / 2$ chinois alors qu'elle est négative $(-3 \mathrm{~mm})$ chez les sujets de race pure Piétrain. 
TABLEAU 3

Performances moyennes des types génétiques dans les comparaisons $n^{\text {ox }} 1$ et 2 (porcs $1 / 2$ chinois).

Means of genetic types in comparisons 1 and 2 (50 p. 100 Chinese pigs).

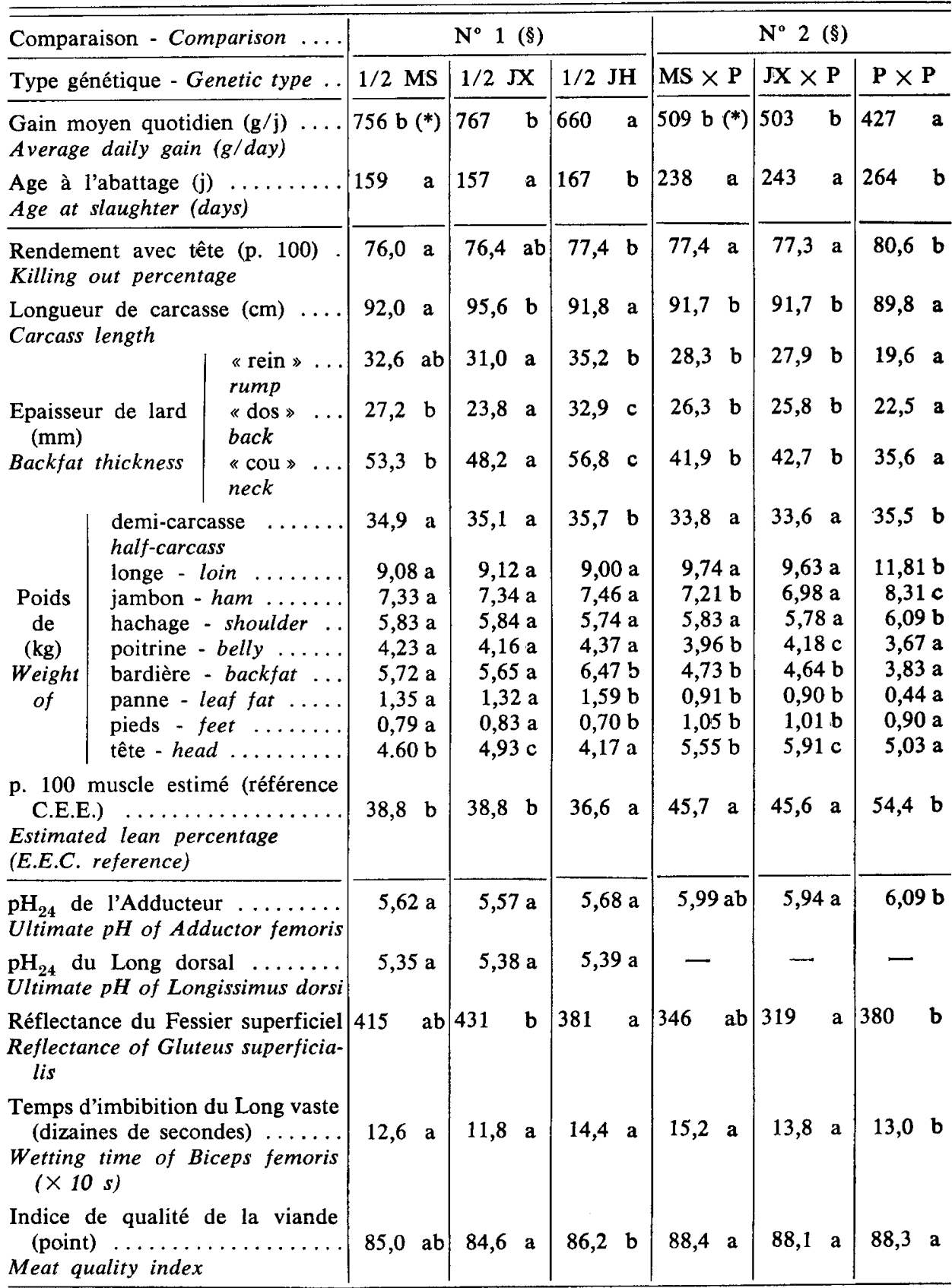

(§) Période de contrôle (test period) : respectivement $29-99 \mathrm{~kg}$ et $27-93 \mathrm{~kg}$ dans les comparaisons $\mathrm{n}^{\circ} 1$ et 2 . Caractères de carcasse ajustés à poids vif d'abattage constant : respectivement 99 et $93 \mathrm{~kg}$ dans les comparaisons $\mathrm{n}^{\circ \mathrm{s}} 1$ et 2 (carcass traits adjusted to a constant slaughter liveweight : 99 and $93 \mathrm{~kg}$ in the comparisons 1 and 2 , respectively).

(*) Dans chaque comparaison, 2 moyennes affectées de la même lettre ne diffèrent pas significativement au seuil de 5 p. 100 (within each comparison, two means with the same letter do not differ at the 5 p. 100 significance level). 
Pour les critères prédicteurs de la qualité technologique de la viande, on relève quelques résultats discordants. Le $\mathrm{pH}$ ultime du muscle Adductor femoris est significativement plus élevé chez les " témoins " que chez les $\mathrm{JX} \times \mathrm{P}$, les MS $\times \mathrm{P}$ occupant une position intermédiaire. Par contre, la viande est significativement plus colorée chez les JX $\times \mathrm{P}$ que chez les $\mathrm{P} \times \mathrm{P}$, les $\mathrm{MS} \times \mathrm{P}$ occupant de nouveau une position intermédiaire. Pour le pouvoir de rétention d'eau, on n'observe pas de différence significative entre types génétiques. Au total, l'effet de la race paternelle est minime pour l'indice de qualité de la viande, qui est une combinaison linéaire de ces différents critères.

\section{B. Porcs $1 / 4$ chinois}

Pour la raison énoncée précédemment, nous avons choisi de présenter seulement les estimées relatives aux effets des types génétiques maternels regroupés en 4 classes (1/2 MS, 1/2 JX, 1/2 JH et européen) sur les critères d'engraissement et de carcasse des produits terminaux issus de verrats LB ou LW. Ce mode de présentation apparaît d'autant plus approprié que l'on n'observe pas d'effet significatif de la race grand-maternelle d'origine européenne (LW ou LF) et que, pour la quasitotalité des variables, il n'y a pas d'interaction significative entre le type génétique de la mère et le sexe du produit.

A une exception près (le temps d'imbibition), l'effet du type génétique maternel est significatif sur les variables étudiées. Les moyennes des moindres carrés pour les 4 types génétiques de mère, ainsi que la signification statistique des différences entre moyennes prises 2 à 2, figurent au tableau 4 .

Vis-à-vis du " témoin " européen, l'âge à l'abattage est avancé de 13 jours chez les $1 / 4$ MS et de 3 jours chez les $1 / 4 \mathrm{JX}$ mais retardé de 6 jours chez les $1 / 4 \mathrm{JH}$. Toutefois, lors du contrôle de croissance lui-même (de 26 à $100 \mathrm{~kg}$ ), le gain moyen quotidien est inférieur respectivement de 28,64 et $118 \mathrm{~g}$ chez les $1 / 4 \mathrm{MS}$, les $1 / 4 \mathrm{JX}$ et les $1 / 4 \mathrm{JH}$. L'indice de consommation entre 26 et $100 \mathrm{~kg}$ est augmenté respectivement de $0,23,0,34$ et 0,39 point chez les $1 / 4 \mathrm{MS}$, les $1 / 4 \mathrm{JX}$ et les $1 / 4 \mathrm{JH}$, par rapport aux «témoins». Notons aussi que les porcs $1 / 4 \mathrm{MS}$ se distinguent par une consommation journalière d'aliment plus forte.

Les animaux 1/4 JH ont un rendement à l'abattage significativement plus élevé que les «témoins" $(+1,1$ point) et que les animaux $1 / 4 \mathrm{JX}$ et $1 / 4 \mathrm{MS}$ (respectivement $+1,0$ et $+1,5$ point). Sur le plan de la longueur, les types génétiques se répartissent en 2 catégories, les carcasses plus longues étant observées chez les porcs $1 / 4 \mathrm{JX}$ et « témoins».

Nous ne commenterons pas en détail les résultats concernant les poids des morceaux de la découpe normalisée de la carcasse qui figurent au tableau 4. Le pourcentage estimé de maigre dans la carcasse est significativement plus faible $(P<0,001)$ chez les $1 / 4$ chinois que chez les "témoins ": la réduction va de 3,5 points chez les $1 / 4$ MS à 5,2 points chez les $1 / 4 \mathrm{JH}$. L'épaisseur du lard dorsal mesurée en 3 points de la carcasse est significativement plus élevée chez les $1 / 4$ chinois que chez les «témoins", sauf pour la mesure au "dos" chez les 1/4 JX; pour l'épaisseur moyenne de lard aux 3 sites, l'avantage des porcs européens est respectivement de $1,7,2,9$ et $5,8 \mathrm{~mm}$ vis-à-vis des porcs $1 / 4 \mathrm{JX}, 1 / 4 \mathrm{MS}$ et $1 / 4 \mathrm{JH}$. 


\section{TABLEAU 4}

Performances moyennes des types génétiques dans les comparaisons $n^{o s} 3$ et 4 (porcs $1 / 4$ chinois) (\$).

Means of genetic types in comparisons 3 and 4 (25 p. 100 Chinese pigs).

\begin{tabular}{|c|c|c|c|c|}
\hline Type génétique - Genetic type & $1 / 4 \mathrm{MS}$ & $1 / 4 \mathrm{JX}$ & $1 / 4 \mathrm{JH}$ & européen \\
\hline $\begin{array}{l}\text { Gain moyen quotidien }(\mathrm{g} / \mathrm{j}) \\
\text { Average daily gain }(\mathrm{g} / \text { day })\end{array}$ & 790 & $754 \quad b$ & 700 & 818 \\
\hline $\begin{array}{l}\text { Age à l'abattage }(\mathrm{j}) \\
\text { Age at slaughter (days) }\end{array}$ & 162 & 172 & 181 & 175 \\
\hline $\begin{array}{l}\text { Consommation journalière d'aliment }(\mathrm{kg} / \mathrm{j})(\text { a) } \ldots \\
\text { Daily food consumption }(\mathrm{kg} / \text { day })\end{array}$ & $2,64 \mathrm{~b}$ & $2,48 \mathrm{ab}$ & $2,42 \mathrm{a}$ & 2,45 a \\
\hline $\begin{array}{l}\text { Indice de consommation }(\mathrm{kg} \text { aliment } / \mathrm{kg} \text { gain) (a) } \\
\text { Food conversion ratio }(\mathrm{kg} \text { feed } / \mathrm{kg} \text { gain) }\end{array}$ & $3,63 \mathrm{~b}$ & $3,74 \mathrm{bc}$ & $3,79 \mathrm{c}$ & $3,40 \mathrm{a}$ \\
\hline $\begin{array}{l}\text { Rendement avec tête (p. 100) } \ldots \ldots \ldots \ldots \cdots \cdots \\
\text { Killing out percentage }\end{array}$ & 77,9 a & 78,4 b & 79,4 c & 78,3 ab \\
\hline $\begin{array}{l}\text { Longueur de carcasse }(\mathrm{cm}) \ldots \ldots \ldots \ldots \ldots \ldots \\
\text { Carcass length }\end{array}$ & 95,8 a & 97,9 b & 95,3 a & 97,0 b \\
\hline \begin{tabular}{l|l} 
Epaisseur de lard $(\mathrm{mm})$ & $\begin{array}{l}\text { "rein } »-\operatorname{rump} \ldots \\
\text { Backfat thickness }\end{array}$ \\
«dos $»-$ back $\ldots . .$. \\
«cou $»-$ neck $\ldots . .$.
\end{tabular} & $\begin{array}{ll}31,1 & \mathrm{c} \\
27,3 & \mathrm{~b} \\
48,0 & \mathrm{~b}\end{array}$ & $\begin{array}{ll}29,9 & b \\
25,6 & a \\
47,3 & b\end{array}$ & $\begin{array}{ll}32,4 & \mathrm{c} \\
30,0 & \mathrm{c} \\
52,9 & \mathrm{c}\end{array}$ & $\begin{array}{ll}27,8 & \text { a } \\
25,1 & \text { a } \\
44,9 & \text { a }\end{array}$ \\
\hline $\mid \begin{array}{l}\text { demi-carcasse }- \text { half-carcass } \ldots \\
\text { longe - loin } \ldots \ldots \ldots \ldots \ldots \ldots \\
\text { jambon - ham } \ldots \ldots \ldots \ldots \ldots \\
\text { hachage - shoulder } \ldots \ldots \ldots \ldots \\
\text { poitrine }- \text { belly } \ldots \ldots \ldots \ldots \\
\text { bardière }- \text { backfat } \ldots \ldots \ldots \ldots \\
\text { panne }- \text { leaf fat } \ldots \ldots \ldots \ldots \\
\text { pieds }- \text { feet } \ldots \ldots \ldots \ldots \ldots \ldots \\
\text { tête }- \text { head } \ldots \ldots \ldots \ldots \ldots\end{array}$ & $\begin{array}{r}36,0 \mathrm{a} \\
10,47 \mathrm{a} \\
8,39 \mathrm{~b} \\
5,59 \mathrm{~b} \\
4,32 \mathrm{a} \\
5,21 \mathrm{~b} \\
0,92 \mathrm{~b} \\
1,01 \mathrm{c} \\
4,06 \mathrm{~b}\end{array}$ & $\begin{array}{l}36,1 \mathrm{ab} \\
10,54 \mathrm{ab} \\
8,28 \mathrm{a} \\
5,60 \mathrm{~b} \\
4,39 \mathrm{~b} \\
5,24 \mathrm{~b} \\
0,97 \mathrm{~b} \\
1,00 \mathrm{c} \\
4,24 \mathrm{c}\end{array}$ & $\begin{array}{l}36,9 \mathrm{c} \\
10,65 \mathrm{~b} \\
8,37 \mathrm{ab} \\
5,49 \mathrm{a} \\
4,48 \mathrm{~b} \\
5,81 \mathrm{c} \\
1,19 \mathrm{c} \\
0,85 \mathrm{a} \\
3,81 \mathrm{a}\end{array}$ & $\begin{array}{l}36,3 \mathrm{~b} \\
11,20 \mathrm{c} \\
8,86 \mathrm{c} \\
5,51 \mathrm{ab} \\
4,23 \mathrm{a} \\
4,72 \mathrm{a} \\
0,76 \mathrm{a} \\
0,94 \mathrm{~b} \\
3,88 \mathrm{a}\end{array}$ \\
\hline $\begin{array}{l}\text { p. } 100 \text { muscle estimé (référence C.E.E.) } \ldots \ldots \\
\text { Estimated lean percentage (E.E.C. reference) }\end{array}$ & $45,6 \mathrm{~b}$ & $45,1 \quad b$ & 43,9 a & 49,1 c \\
\hline $\begin{array}{l}\mathrm{pH}_{2+} \text { du jambon }(b) \ldots \ldots \ldots \ldots \ldots \ldots \ldots \ldots \ldots \\
\text { Ultimate } p H \text { of ham }\end{array}$ & $5,66 \mathrm{~b}$ & $5,62 \mathrm{a}$ & 5,61 a & $5,61 \mathrm{ab}$ \\
\hline $\begin{array}{l}\mathrm{PH}_{24} \text { du Long dorsal } \ldots \ldots \ldots \ldots \ldots \ldots \ldots \ldots \\
\text { Ultimate } p H \text { of } L . \text { dorsi }\end{array}$ & $5,56 \mathrm{~b}$ & $5,53 \mathrm{ab}$ & $5,50 \mathrm{a}$ & 5,49 a \\
\hline $\begin{array}{l}\text { Réflectance du jambon }(b) \ldots \ldots \ldots \ldots \ldots \ldots \ldots \\
\text { Reflectance of ham }\end{array}$ & 369 & 366 & 370 & 390 \\
\hline $\begin{array}{l}\text { Temps d'imbibition du jambon (dizaines de se- } \\
\text { condes) }(b) \ldots \ldots \ldots \ldots \ldots \ldots \ldots \ldots \ldots \ldots \ldots \\
\text { Wetting time of ham }(\times 10 \text { s) }\end{array}$ & 10,9 a & 11,2 a & 10,6 a & 9,9 a \\
\hline $\begin{array}{l}\text { Indice de qualité de la viande (point) } \ldots \ldots \ldots \\
\text { Meat quality index }\end{array}$ & 86,3 a & 86,1 a & 86,0 a & 85,8 a \\
\hline
\end{tabular}

(\$) Période de contrôle (test period) : $26-100 \mathrm{~kg}$.

Caractères de carcasse ajustés à poids vif d'abattage constant : $100 \mathrm{~kg}$ (carcass traits adjusted to a constant slaughter liveweight : $100 \mathrm{~kg}$ ).

(a) Variables mesurées seulement dans la comparaison $\mathrm{n}^{\circ} 3$ (traits recorded only in comparison 3).

(b) $\mathrm{pH}_{24} \mathrm{du}$ jambon : moyenne des muscles Adductor femoris, Biceps femoris, Gluteus superficialis (average pH of 3 muscles). Réflectance et temps d'imbibition du jambon : moyennes des muscles Biceps femoris et Gluteus superficialis (average of 2 muscles). 
La supériorité des porcs européens sur les porcs $1 / 4$ chinois pour les critères d'adiposité est du même ordre de grandeur chez les femelles et les mâles castrés. Remarquons toutefois que l'interaction sexe $\times$ type génétique de la mère atteint le seuil de signification de 1 p. 100 pour le poids de longe et de 5 p. 100 pour le poids de muscle estimé dans la carcasse avec tête (fig. 1) : pour ces 2 variables, le désavantage des $1 / 4$ chinois est plus marqué chez les femelles que chez les mâles castrés.
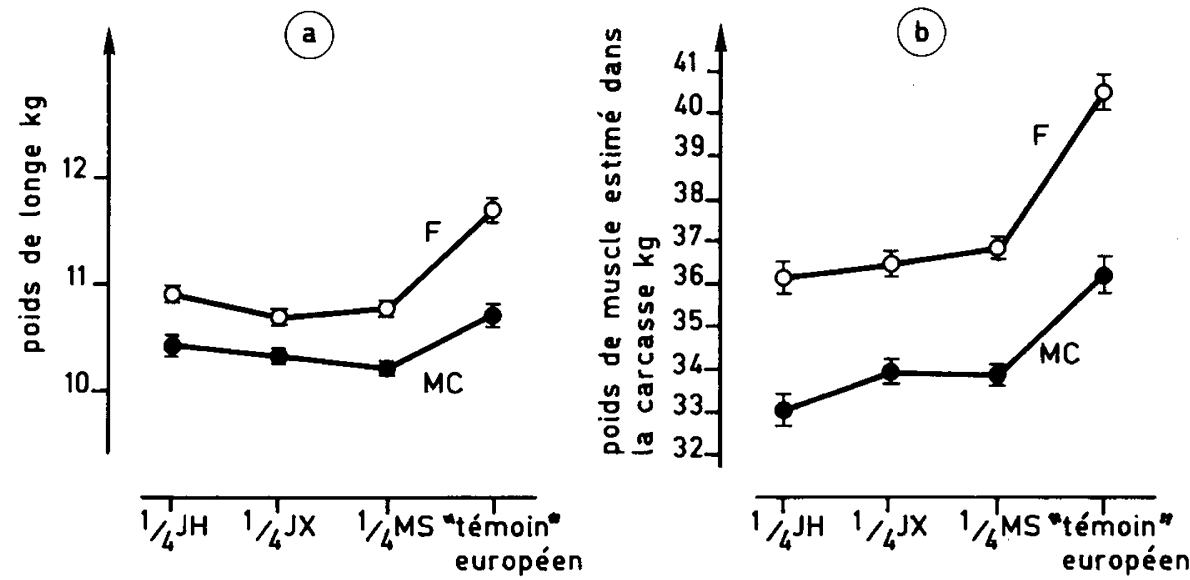

type génétique (genetic type)

Fig. 1

Interaction entre sexe et type génétique pour le poids de longe par $1 / 2$ carcasse (a) et le poids estimé de muscle dans la carcasse entière (b).

Interaction between sex and genetic type in weight of loin in the half-carcass (a) and estimated weight of lean in the entire carcass $(b)$.

F : Femelles - Females.

MC : Mâles castrés - Castrated males.

Les carcasses des $1 / 4 \mathrm{JH}$ sont significativement plus grasses que celles des $1 / 4 \mathrm{MS}$ et des 1/4 JX et présentent un plus faible pourcentage estimé de muscle, en dépit d'un poids de longe légèrement plus élevé. En outre, les $1 / 4 \mathrm{JH}$ semblent présenter un poids de squelette plus faible (d'après les poids de la tête et des pieds) que les $1 / 4$ MS et les 1/4 JX. Ces 2 derniers types génétiques apparaissent, d'une façon générale, très proches l'un de l'autre sur le plan de la composition corporelle.

Les 4 critères de qualité de la viande sont améliorés dans leur ensemble chez les $1 / 4$ chinois comparativement aux " témoins » mais les différences sont rarement significatives. Ainsi, l'augmentation du $\mathrm{pH}$ ultime vis-à-vis des « témoins » n'est significative que chez les $1 / 4 \mathrm{MS}$ dans le muscle Long dorsal. Pour la réflectance, l'écart aux " témoins", dans le sens d'une viande plus colorée, est significatif pour les 3 types de porcs $1 / 4$ chinois, alors que l'augmentation du temps d'imbibition et de l'indice de qualité de la viande observée chez ces derniers n'atteint pas le seuil de signification. 


\section{Discussion}

Soulignons avant toute chose qu'une certaine prudence est recommandée dans l'interprétation des résultats de cette étude et surtout que leur généralisation aux 3 races chinoises concernées serait pour le moins prématurée : il est, en effet, essentiel de garder à l'esprit que l'évaluation de ces races en croisement à 2 ou 3 voies repose sur la descendance d'au plus 3 reproducteurs par race, les fondateurs importés de Chine en 1979.

Il est par ailleurs utile de rappeler que les comparaisons ont été conduites avec une distribution à volonté d'un aliment relativement riche en énergie. L'alimentation ad libitum conduit à une expression maximale des différences de composition corporelle entre types génétiques et donc du désavantage des types génétiques les plus gras (Henry \& Etienne, 1978) : dans l'étude de Davey et al. (1969), une interaction entre le niveau d'apport énergétique et le type génétique (lignées grasse et maigre d'une même race) a été mise en évidence pour le poids de tissu maigre produit, l'effet favorable de la restriction alimentaire étant plus accusé dans la lignée grasse que dans la lignée maigre. Il est vraisemblable que le désavantage de composition corporelle des porcs $1 / 4$ chinois vis-à-vis des «témoins » européens tendrait à se réduire avec un apport restreint d'énergie, par la voie du rationnement.

La hiérarchie établie entre les 3 races pures chinoises sur le plan de la vitesse de croissance et du développement corporel (LEGAULT et al., 1982) est généralement bien respectée à l'occasion des croisements à 2 et 3 voies avec les races européennes. Dès la naissance, les représentants de la race Jinhua accusent un important retard de croissance sur ceux de la race Meishan, la race Jiaxing occupant en général une position intermédiaire. Un avantage notable du Meishan sur le Jiaxing est également observé en Chine de ce point de vue : 77,5 contre $56,5 \mathrm{~kg}$ pour le poids à 240 jours selon ZhaNg et al. (1983). Dans le milieu français, les animaux de race pure chinoise présentent une vitesse de croissance modeste : ainsi, les cochettes Meishan, Jiaxing et Jinhua atteignent respectivement les poids de 66,56 et $47 \mathrm{~kg}$ à l'âge de 5 mois (LEgault et al., 1982). Le niveau satisfaisant des animaux $1 / 2$ chinois pour la vitesse de croissance (tabl. 3) peut s'expliquer par l'existence d'une aptitude spécifique à la combinaison très favorable qui se traduit par un effet d'hétérosis particulièrement élevé. L'effet d'hétérosis sur le gain moyen quotidien n'a pu, dans le cadre de cette étude, être estimé de façon rigoureuse, en l'absence de porcs contemporains des races pures européennes. Il est cependant permis de supposer qu'il est de l'ordre de 15 p. 100, moyenne des valeurs rapportées par ZHANG et al. (1983) dans divers croisements entre races chinoises et races européennes ou nord-américaines. Un effet d'hétérosis de 11 p. 100 a été observé par XU et al. (1983) dans le croisement Large White $\times$ Jiaxing pour le gain moyen quotidien de 26 à $90 \mathrm{~kg}$. Il est à noter que la vitesse de croissance relativement rapide des croisés chinois se manifeste surtout dans le jeune âge mais qu'un ralentissement de la croissance, plus ou moins accentué, survient en fin d'engraissement.

La tendance au «nivellement " des vitesses de croissance par le biais de l'hétérosis n'est pas suffisante pour combler le lourd handicap des races chinoises sur le plan de l'efficacité alimentaire : le désavantage des animaux 1/4 chinois, par rapport aux « témoins " européens, est compris entre 0,23 et 0,39 point d'indice de consom- 
mation. La moindre efficacité alimentaire des porcs $1 / 4$ chinois est due en partie à l'élévation du coût énergétique du gain de poids vif : ce coût est en relation étroite avec le contenu énergétique du gain, lui-même fonction du pourcentage d'énergie fixée sous forme de lipides et donc de l'importance des dépôts gras (HeNRY \& ETIENNE, 1978).

En matière de composition corporelle, les données présentées ici confirment nos propres résultats préliminaires (LEGAULT et al., 1982) et les résultats obtenus en Chine : les races chinoises importées en France ont, dans ce domaine, un « retard génétique " considérable sur les races européennes usuelles. Des 3 races étudiées, le Jinhua a la plus forte teneur en morceaux gras mais aussi le meilleur rendement en carcasse. Le Meishan et le Jiaxing sont, dans l'ensemble, assez proches l'un de l'autre sur le plan de la qualité de carcasse.

Nous présentons sous forme graphique (fig. 2) une tentative de synthèse des résultats des 4 comparaisons pour le rendement et le pourcentage de muscle estimé dans la carcasse. Les moyennes présumées des races Meishan, Jiaxing et Jinhua ont été calculées par extrapolation des résultats observés chez les produits de croisement $1 / 2$ et $1 / 4$ chinois, en faisant l'hypothèse de l'additivité des effets génétiques pour les 2 caractères. Pour le rendement en carcasse (avec tête), le Jinhua, race chinoise la plus grasse, est proche des races européennes à fort développement musculaire (Piétrain, Landrace Belge), alors que le Jiaxing est voisin du Large White et du Landrace Français : la position du Jiaxing s'explique surtout par un poids de tête élevé. Pour le pourcentage de muscle estimé dans la carcasse avec tête (référence C.E.E.), les 3 races pures chinoises se situent approximativement entre 29-30 p. 100 pour le Jinhua et 35-36 p. 100 pour le Meishan, c'est-à-dire à 15 à 20 points de pourcentage au-dessous du Large White. A propos du pourcentage de muscle, il importe de considérer avec précaution les estimations rapportées dans cet article. L'équation de prédiction utilisée, due à Pommerer \& Naveau (1979), a été établie d'après les résultats de dissection d'un échantillon de porcs des races européennes (teneur en muscle comprise entre 40 et 60 p. 100). Son application à un matériel génétique d'un type très différent, "sortant " nettement (au moins pour les porcs $1 / 2$ chinois) de la gamme des teneurs en muscle donnée ci-dessus, est une extrapolation qui comporte un risque de biais, comme le souligne MACNeIL (1983). Nos chiffres de pourcentage de muscle pour les 3 races chinoises sont donc à prendre comme des ordres de grandeur. Remarquons cependant que l'écart trouvé ici entre Jiaxing et Large White pour le pourcentage de muscle est voisin de l'écart de 17 points rapporté dans une étude chinoise concernant ces deux races (Xu et al., 1983).

Pour bien apprécier les différences très importantes de composition tissulaire de la carcasse entre races chinoises et races européennes, il faut tenir compte d'une caractéristique originale des races chinoises, à savoir l'importance relative nettement accrue du poids de peau. Les différences d'épaisseur de lard dorsal et de poids de bardière entre porcs $1 / 4$ chinois et porcs européens ne proviennent pas seulement de l'accroissement du dépôt sous-cutané de gras dorsal mais aussi de l'accroissement de l'épaisseur et du poids de peau. Les résultats de dissection rapportés par LiaO MingShOU (1981) et ZHAO et al. (1983) sont éloquents à ce sujet. Dans l'étude de ZHAO et al. (1983), l'épaisseur de la peau est respectivement de 6,7 et 4,1 $\mathrm{mm}$ et le pourcentage de peau dans la carcasse (sans tête, pieds et panne) est respectivement de 16,6 et 9,2 p. $100 \mathrm{chez}$ des porcs de race pure Meishan et des porcs Large White $\times$ Meishan abattus à $90 \mathrm{~kg}$. Rappelons que, dans les races européennes usuelles, le pour- 
centage de peau, rapporté à un poids de carcasse défini comme ci-dessus, est de l'ordre de 4 p. 100 chez l'animal de 90-95 kg (Desmoulin \& Pommeret, 1974 ; Evans \& KEMPSTER, 1979).

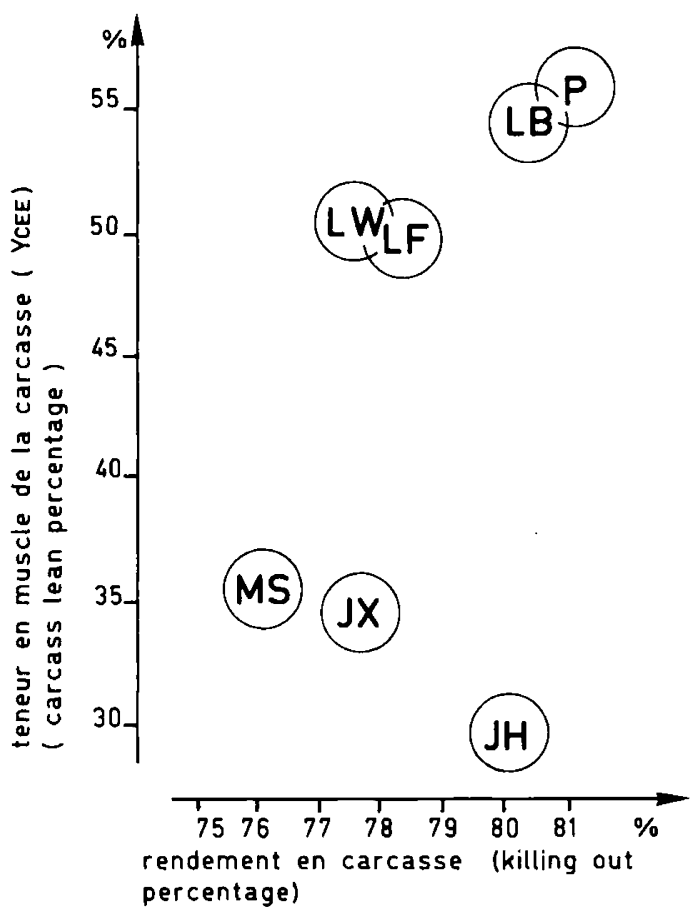

FIG. 2

Position présumée des 3 races pures chinoises, vis-à-vis des principales races exploitées en France, pour le rendement en carcasse et le pourcentage de muscle estimé dans la carcasse (référence C.E.E.).

Presumed position of the 3 Chinese pure breeds, as compared to the main breeds used in France, in killing out percentage and estimated carcass lean percentage (E.E.C. reference).

Une autre particularité des races chinoises qui mérite d'être notée a trait au " profil » de la couche de lard dorsal sur la fente saggitale de la carcasse. La figure 3 représente la position comparée des races chinoises Meishan et Jiaxing vis-à-vis des races européennes (Large White ou Landrace Français et Piétrain : voir Dumont \& Roy, 1974) pour l'épaisseur de lard au « rein», au «dos» et au «cou». Pour la différence d'épaisseur de lard entre les 2 premiers sites de mesure $(d=$ "rein "dos »), il existe un gradient marqué entre races : $d$ est négatif dans la race la plus maigre (Piétrain), proche de zéro ou légèrement positif dans la race à adiposité moyenne (Large White), et nettement positif dans les races chinoises, à forte adiposité. La figure 3 montre incidemment que l'épaisseur de lard au " rein » est un meilleur indicateur du pourcentage de morceaux gras que la mesure au " dos ». 

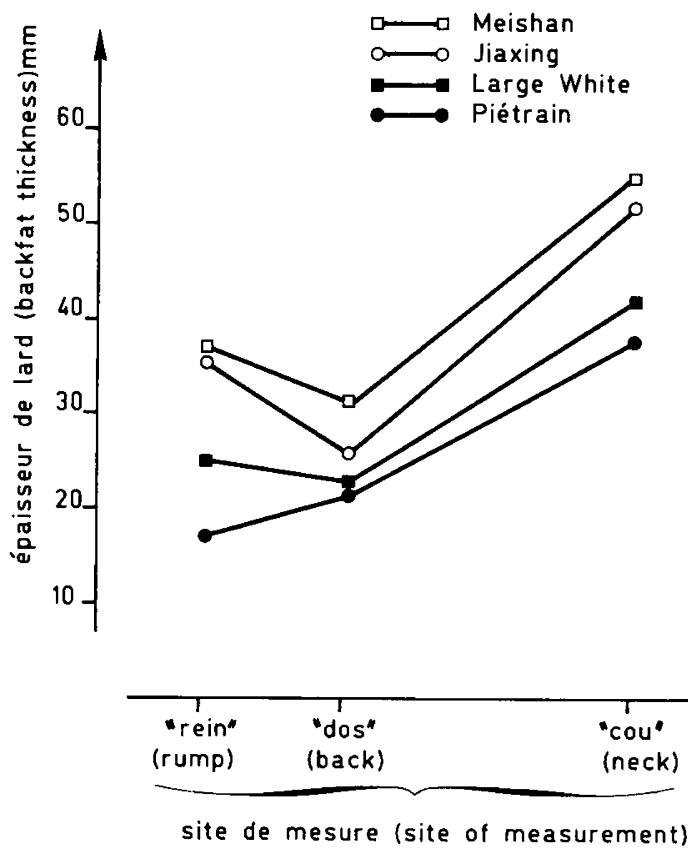

Fig. 3

Profil longitudinal de la couche de lard dorsal dans les races pures Meishan, Jiaxing, Large White et Piétrain.

"Side-view» of the backfat layer in the Meishan, Jiaxing, Large White and Pietrain pure breeds.

L'interaction sexe $\times$ type génétique trouvée ici pour le poids de longe et pour l'estimateur du poids total de tissu maigre dans les comparaisons concernant les porcs $1 / 4$ chinois va dans le sens d'un phénomène noté par plusieurs auteurs lors de comparaisons entre types génétiques, en particulier quand l'aliment est distribué à volonté (Bereskin \& Davey, 1976 ; Sellier, 1977 ; Davey \& Bereskin, 1978) : l'avantage de composition corporelle du type maigre (ici le «témoin " européen) sur le type gras (ici les porcs $1 / 4$ chinois) est plus accusé chez les femelles que chez les mâles castrés.

Sur l'ensemble des comparaisons présentées ici, il ne semble pas exister de différences vraiment notables entre les 3 races chinoises étudiées pour les critères prédicteurs de l'aptitude à la salaison et à la cuisson de la viande. Un avantage significatif du Jinhua sur le Jiaxing est trouvé dans la comparaison $\mathrm{n}^{\circ} 1$ (1/2 chinois) pour l'indice de qualité de la viande mais il n'est pas confirmé dans la comparaison entre porcs $1 / 4$ Jinhua et $1 / 4$ Jiaxing.

Par ailleurs, il ressort des comparaisons $n^{0 s} 3$ et 4 que les races chinoises étudiées ne diffèrent guère du Large White et du Landrace Français pour les critères de qualité technologique de la viande $(\mathrm{pH}$ ultime, couleur, pouvoir de rétention d'eau). Les résultats vont toutefois dans le sens d'un indice de qualité de la viande 
très légèrement supérieur chez les porcs $1 / 4$ chinois, comparés aux " témoins \# européens; l'effet le plus marqué concerne la couleur significativement plus prononcée de la viande chez les porcs $1 / 4$ chinois.

Si les races chinoises ne se distinguent pas de façon vraiment marquée des races européennes de type Large White ou Landrace sur le plan des qualités technologiques de la viande, il n'en est pas de même sur le plan des qualités organoleptiques, d'après les premiers résultats obtenus en France (dans le cadre de la comparaison $n^{\circ} 2$ ) et rapportés par ailleurs (Touraille et al., 1983). Il a été montré, lors d'un test de dégustation, que les porcs Meishan $\times$ Piétrain et Jiaxing $\times$ Piétrain sont jugés nettement supérieurs aux porcs de race pure Piétrain en ce qui concerne la jutosité, la flaveur et surtout la tendreté de rôtis prélevés dans la longe. Il est vraisemblable qu'un taux de lipides supérieur des viandes de croisés chinois est à l'origine de cet avantage : l'augmentation de la teneur en gras intramusculaire est en effet connue pour améliorer la tendreté, la jutosité et, jusqu'à une certaine limite, la flaveur de la viande.

Dans le domaine des qualités technologiques ou organoleptiques de la viande, les données bibliographiques relatives aux races porcines chinoises sont encore fragmentaires. Des résultats recueillis en Chine indiquent toutefois que, pour le rendement à la cuisson de la viande, la supériorité des races chinoises est plus forte que ne le laissent prévoir les différences minimes que nous rapportons ici pour l'indice de qualité de la viande. Ainsi, Xu et al. (1983) ont trouvé que les porcs de race Jiaxing sont supérieurs aux porcs de race Large White $(+4,6$ points de pourcentage) pour le rendement à la cuisson de la longe. Dans une comparaison entre porcs de race pure Meishan et porcs issus de croisements $\mathrm{F}_{1}$ entre le Meishan et diverses races européennes (Large White, Landrace) ou nord-américaines (Duroc, Hampshire), ZHaO et al. (1983) ont également observé pour le même caractère un avantage des porcs Meishan, allant jusqu'à 2,2 points de pourcentage vis-à-vis des porcs Hampshire $\times$ Meishan.

\section{Conclusion}

Sur la base des pondérations économiques les plus récentes retenues pour le contrôle des produits terminaux en France (ANONYME, 1983), soit 0,135 franc par gramme de gain moyen quotidien, $-121,5$ francs par point d'indice de consommation, 8 francs par $\mathrm{kg}$ de muscle dans la carcasse et 3,8 francs par point d'indice de qualité de la viande, les produits terminaux $1 / 4$ Meishan, $1 / 4$ Jiaxing et 1/4 Jinhua présentent dans le cadre de cette étude une marge brute par porc réduite respectivement de 54, 74 et 93 francs par rapport aux " témoins " européens.

La réduction du prix de revient unitaire des porcelets issus de truies $1 / 2$ Meishan et 1/2 Jiaxing, comparées à des truies européennes, avait été située entre 40 et 60 francs dans une première estimation (Legault \& CARITEZ, 1983). Une estimation plus récente (LEGAULt et al., 1984), faite sur la base d'un coût d'entretien annuel de la truie de 3900 francs (TEFFENE, 1983), situe cette réduction entre 53 et 62 francs. Le bilan économique global est donc voisin de l'équilibre pour les truies $1 / 2 \mathrm{Meishan}$ et légèrement défavorable pour les truies $1 / 2$ Jiaxing. Cependant, il pourrait être amélioré en faveur des femelles $1 / 2$ chinoises dans la mesure où l'on tient compte de leur aptitude à une mise à la reproduction plus précoce. Selon NoguERA \& GuEBLEZ (1984), une première mise bas avancée d'un mois peut en effet abaisser le prix 
de revient du porcelet au sevrage d'environ 10 francs. Toutefois, ce dernier aspect, ainsi que la conduite de truies dont la productivité numérique est améliorée d'au moins 30 p.100, réclament de la part de l'éleveur une grande technicité. Si l'amélioration de la productivité des truies par le recours à certaines races chinoises peut être considérée comme un potentiel à la portée des meilleurs éleveurs, la dégradation de l'efficacité alimentaire et de la qualité de la carcasse qui en découle chez les produits peut être considérée comme une certitude pour l'ensemble des éleveurs.

L'ensemble des résultats confirme nos premières observations sur la valeur comparée, en croisement avec les races occidentales, des 3 races chinoises. Les performances des animaux croisés Jinhua sont significativement inférieures à celles de leurs homologues Meishan et Jiaxing sur presque tous les plans : reproduction, croissance et composition corporelle. Toutefois, l'extrême exiguité des échantillons de reproducteurs fondateurs ( 1 mâle et 2 femelles par race) interdit toute extrapolation hâtive de ces résultats aux 3 races d'origine dans leur ensemble. L'expérimentation en France se poursuit uniquement sur le Meishan et le Jiaxing dont les performances en croisement sont très proches en matière de prolificité, de composition corporelle et de qualité de la viande, alors qu'un avantage significatif du Meishan est enregistré pour la croissance et le développement corporel.

La valorisation en élevage intensif de l'aptitude à la reproduction exceptionnelle des races chinoises du type Taihu, dont le Meishan et le Jiaxing sont 2 des représentants, exige encore un complément d'expérimentation visant notamment à l'estimation rigoureuse des paramètres de croisement (hétérosis direct et maternel, effets maternels et grand-maternels, effets de recombinaison, ...) et à une meilleure évaluation de leurs particularités biologiques, compte tenu de leur éloignement génétique assez considérable vis-à-vis des races occidentales.

Reçu le 7 mars 1984. Accepté le 7 juin 1984.

\section{Références bibliographiques}

ANONYME, 1983. Résultats du $10^{\circ}$ test d'évaluation des performances d'engraissement, de carcasse et de qualité de viande des produits terminaux des schémas de sélection et croisement. Techni-Porc, 6 (6), 47-65.

Bereskin B., Davey R.J., 1976. Breed, line, sex and diet effects and interactions in swine carcass traits. J. Anim. Sci., 42, 43-51.

Cheng P.L., 1983. A highly prolific pig breed of China. The Taihu pig. Pig New's and Information, 4, 407-416.

Cheng P.L., 1984. A highly prolific pig breed of China. The Taihu pig : parts III and IV. Pig News and Information, 5, 13-18.

DaVeY R.J., BereskiN B.. 1978. Genetic and nutritional effects on carcass chemical composition and organ weights of market swine. J. Anim. Sci., 46, 992-1000.

DaveY R.J., Morgan D.P., Kincaid C.M., 1969. Response of swine selected for high and low fatness to a difference in dietary energy intake. J. Anim. Sci., 28, 197-203.

Desmoulin B., Pommeret P., 1974. Références de composition anatomique et critères de classification des carcasses de porcs femelles des types Landrace Français, Landrace Belge et Piétrain. Journées de la Recherche Porcine en France, Paris, 20-22 février 1974, 221-232, Institut Technique du Porc, Paris.

Dumont B.L., Roy G., 1974. Variation de la forme du dos et du rein de trois races (Landrace Français, Landrace Belge et Piétrain). Journées de la Recherche Porcine en France, Paris, 20-22 février 1974, 241-246, Institut Technique du Porc, Paris. 
Evans D.G., Kempster A.J., 1979. The effects of genotype, sex and feeding regimen on pig carcass development. 1. - Primary components, tissues and joints. J. Agric. Sci., 93, 339-347.

Gianola D., Legault C., Caritez J.C., 1982. Evaluation of Chinese swine germplasm in France : preliminary results. University of Illinois Pork Industry Conference, UrbanaChampaign, 2-3 décembre 1982, 39-55.

Henry Y. ETIENNE M., 1978. Alimentation énergétique du porc. 10* Journées de la Recherche Porcine en France, Paris, 1-3 février 1978, 119-166, Institut Technique du Porc, Paris.

Jacquet B., Sellier P., Runavot J.P., Brault D., Houix Y., Perrocheau C., Gogué J., Boulard J., 1984. Prédiction du rendement technologique de la fabrication du "jambon de Paris» à l'aide de mesures prises à l'abattoir. $16^{\text {es }}$ Journées de la Recherche Porcine en France, Paris, 1-3 février 1984, 49-58, Institut Technique du Porc, Paris.

Legault C., Caritez J.C., 1983. L'expérimentation sur le porc chinois en France. 1. - Performances de reproduction en race pure et en croisement. Génét. Sél. Evol., 15, 225-240.

Legault C., Caritez J.C., Gruand J., Bidanel J.P., 1984. Le point de l'expérimentation sur les races chinoises en France : «reproduction» et "production》. $16^{s s}$ Journées de la Recherche Porcine en France, Paris, 1-3 février 1984, 481-494, Institut Technique du Porc, Paris.

Legault C., Caritez J.C., Gruand J., Sellier P., 1982. Premier bilan de l'expérimentatation sur le porc chinois en France. 3. - Croissance et composition corporelle en croisement à deux ou trois voies. $14^{*}$ Journées de la Recherche Porcine en France, Paris, 3-4 février 1982, 143-150, Institut Technique du Porc, Paris.

Liao Mingshou, 1981. (Experiments on crossbreeding using the Taihu pig as the female parent). Hubei Nongye Kexue, 1981, n" 2, 31-34 (Anim. Breed. Abstr., 49, 843, Abstr.).

MACNEIL M.D., 1983. Choice of a prediction equation and the use of the selected equation in subsequent experimentation. J. Anim. Sci., 57, 1328-1336.

Noguera J.L., Gueblez R., 1984. Incidence de l'âge à la première mise bas et de la taille de la première portée sur la carrière de la truie. $16^{e s}$ Journées de la Recherche Porcine en France, Paris, 1-3 février 1984, 135-144, Institut Technique du Porc, Paris.

Ollivier L., 1970. L'épreuve de la descendance chez le porc Large White français de 1953 à 1966. I. - Analyse de la variation. Ann. Génét. Sél. anim., 2, 311-324.

Pommeret P., Naveau J., 1979. Composition anatomique de la carcasse : la découpe parisienne normalisée (D.P.N.). Institut Technique du Porc, Centre Expérimental de Sélection Porcine de Maxent, rapport 79-06, 14 pages ronéotypées.

SEllier P., 1977. Valeur en croisement de verrats Large White et Piétrain. Influence du poids d'abattage. Journées de la Recherche Porcine en France, Paris, 2-3 février 1977, 85-89, Institut Technique du Porc, Paris.

Sellier P., Monin G., Houix Y., Dando P., 1984. Qualité de la viande de quatre races porcines : relations avec la sensibilité à l'halothane et l'activité créatine phosphokinase plasmatique. $16^{\text {es }}$ Journées de la Recherche Porcine en France, Paris, 1-3 février 1984, 65-74, Institut Technique du Porc, Paris.

Teffene O., 1983. Economie de la conduite d'un troupeau de truies. L'Eleveur de Porcs, 150, $65-70$.

Touraille C., Monin G., Legault C., 1983. Qualités organoleptiques des viandes de porcs croisés Piétrain/Chinois. $15^{\mathrm{s} s}$ Journées de la Recherche Porcine en France, Paris, 2-3 février 1983, 215-218, Institut Technique du Porc, Paris.

Wu J.S., Zhang W.C., 1982. Genetic analysis of some Chinese breeds as a resource for world hog improvement. 2nd World Congress on Genetics Applied to Livestock Production, Madrid, 4-8 octobre 1982, 8, 593-600, Editorial Garsi, Madrid.

Xu S.Q., Hong Q.J., Wang S.H.; Cheng Z.W., Zheng K.Z., 1983. Studies on the fattening performance and the meat-fat quality of Jiaxing Black pigs (en chinois). Colloque Franco-Chinois sur l'Elevage Porcin, Shanghai, 22-24 août 1983, 8 pages ronéotypées.

Zhang W.C.; Wu J.S., ReMPEL W.E., 1983. Some performance characteristics of prolific breeds of pigs in China. Livest. Prod. Sci., 10, 59-68.

Zhao Z.L., Zhu H.S., Zhang Y., Zhou S., Chen Z., Ge Y., Jiang P.L., 1983. A study on the improvement of lean content through crossbreeding of the Meishan pig (en chinois). Colloque Franco-Chinois sur l'Elevage Porcin, Shanghai, 22-24 aôt 1983, 6 pages ronéotypées. 